\title{
Functional MRI in pre-surgical planning: case study and cautionary notes
}

\author{
B S Spottiswoode, PhD \\ MRC/UCT Medical Imaging Research Unit, Department of Human Biology, University of Cape Town, and Division of Radiology, Stellenbosch University
}

\section{S Du Plessis, MB ChB}

Division of Psychiatry, Stellenbosch University

\section{A Gretschel, MB ChB, M Med (Neurosurg)}

Division of Neurosurgery, Stellenbosch University

\author{
J Lotz, MA, MSC, FRCR \\ Division of Radiodiagnosis, Stellenbosch University
}

Corresponding author: S du Plessis (stefandup@sun.ac.za)

Background. Since its inception almost 20 years ago, functional magnetic resonance imaging (fMRI) has greatly advanced our knowledge of human brain function. Although the clinical applications of fMRI are still limited, there have recently been encouraging advances for its use in pre-operative functional cortical mapping to identify potentially eloquent areas prior to neurosurgery.

Objectives. We explore the potential use of this emerging technique by presenting a neurosurgical case study, as performed at the Cape Universities Brain Imaging Centre (CUBIC), Tygerberg, Cape Town. We conclude with a brief summary of the potential pitfalls of this technique, as well as cautionary guidelines based on our experience.

Methods and results. A 22-year-old male patient from Tygerberg Hospital underwent the successful resection of an anaplastic astrocytoma after fMRI presurgical planning at our facility. The subject was able to leave the ward unassisted.

Conclusion. If consideration is given to the many limitations of this emerging technique, fMRI can be useful in aiding the neurosurgeon in pre-operative planning of his surgical approach.

\section{S Afr J Rad 2012;16(3):107-110. DOI:10.7196/SAJR.709}

Functional magnetic resonance imaging (fMRI) is an MRI technique capable of estimating regional brain activity during a predefined cognitive task. The number of publications using fMRI has increased exponentially ${ }^{1}$ since the technique was first introduced over 20 years ago. ${ }^{2}$ A PubMed search using the keywords 'fMRI OR ('functional MRI')' at the time of writing, identified 31176 results, with 28882 of these having been published in 2011. Although fMRI has contributed significantly to our understanding of neurocognitive function, the number of resulting clinical applications has been limited. Currently, the most promising direct clinical application is in pre-surgical planning, where fMRI is used to identify the spatial relationship between important functional areas in the brain and the area to be resected. ${ }^{3}$ The fMRI results can thus influence the entry point and trajectory of the surgical intervention to avoid damaging eloquent cortical areas.
These are cortical areas in which injury causes symptomatic cognitive or motor deficits and therefore, if avoided, could greatly influence surgical outcome.

The basis of fMRI is that deoxygenated blood is magnetic whereas oxygenated blood is not. The presence of deoxyhaemoglobin in the tissue being imaged therefore perturbs the magnetic field, resulting in a signal loss as measured by T2*-weighted MR imaging. Performing a task in the MRI scanner causes increased neuronal activity, resulting in increased energy utilisation at the synapse, and consequently local increases in blood flow to the brain regions that are recruited during the task. This characteristic is known as the blood oxygen level dependent (BOLD) effect. The signal change measured by fMRI is consequently not a direct measure of neuronal activity, but rather a combination of flow, metabolism, blood volume and blood oxygenation. This signal change is very small, in the order of $1-2 \%$ of the total signal. fMRI scans typically involve alternating periods of rest and activity over a total scan period of 5 - 10 minutes, to improve the sensitivity of the technique. The colourful activation patterns associated with fMRI indicate the statistical likelihood that the regional blood flow is synchronous with the predefined periods of rest and activity.

The most commonly used fMRI tasks for neurosurgical planning are tactile, motor, language and visual. ${ }^{4}$ Active motor tasks are the most robust and established, although it is interesting to note that recent studies have also reliably identified motor regions using passive tasks ${ }^{5}$ and even at rest using functional connectivity fMRI. ${ }^{6}$ Language tasks can be used to determine speech dominance, ${ }^{7}$ although mixed results have been reported for preoperative mapping. ${ }^{8}$ Even auditory tasks provide surprisingly robust results given the inherent MRI scanner acoustic noise. ${ }^{9,10}$

All modern commercial MRI systems offer functional MRI sequences and data processing packages. These fMRI results are limited to the grey matter, but they can be integrated with diffusion tensor imaging (DTI), which is an MRI technique for assessing white matter integrity ${ }^{11}$ and for tracking white matter fiber bundles. ${ }^{12}$ A number of vendors also provide stereotactic planning systems which integrate fMRI (and DTI) results for surgical planning. 


\section{Objectives}

The interpretation of fMRI results is complex and the technique has still not been adopted as a mainstream clinical tool, even in the developed world. In South Africa, the use of fMRI is not yet officially recognised as a diagnostic radiological technique, yet a number of radiology practices have obtained fMRI capability in anticipation of this. This report presents a surgical planning fMRI case study performed at the Cape Universities Brain Imaging Centre (CUBIC) in Cape Town, and discusses the limitations and some cautionary measures to consider when processing and interpreting the fMRI data.

\section{Methods}

A 22-year-old male patient presented to the Emergency Department at Tygerberg Hospital with a history of new-onset focal seizures with secondary generalisation. The patient admitted to alcohol and cannabis use. He was otherwise well and no further relevant history was obtained; on examination he was neurologically intact.

An MRI scan performed on the 1.5 Tesla system (Siemens MAGNETOM Symphony) at Tygerberg Hospital showed a T1-hypointense, T2-hyperintense well-circumscribed lesion with no surrounding vasogenic oedema in the left fronto-parietal area. There was minimal heterogenous contrast enhancement.

The proximity of the tumour to the primary motor cortex was of particular concern, so the patient was referred to CUBIC for an fMRI scan that was deemed crucial to establish safe resection margins and avoid injury to these eloquent cortical areas during surgery.

The patient was scanned on a 3 Tesla MRI (Siemens MAGNETOM Allegra). A sagittal T1-weighted 3D MPRAGE image was acquired, followed by a DTI sequence with diffusion-weighted volumes in 12 directions and $b=1000 \mathrm{~s} / \mathrm{mm}^{2}$. fMRI motor tasks were performed separately for the left and right side. Thirty-second periods of activity were alternated with equal periods of rest for the duration of the scan. For the lower limb, both the ankle and toes were flexed and extended repeatedly. For the upper limb, elbow and wrist flexion and extension was performed repeatedly while tapping consecutive forefingers against the thumb. The patient was requested to keep his upper arm as stationary as possible to minimise motion artifacts. The identical $\mathrm{T} 2^{*}$-weighted EPI sequence was run for all $4 \mathrm{fMRI}$ tasks, with the following parameters: $\mathrm{TR}=2000 \mathrm{~ms}, \mathrm{TE}=30 \mathrm{~ms}$, slices $=34$, spatial resolution $=3.8 \times 3.8 \times 3.5$ $\mathrm{mm}^{3}$, matrix size $=64 \times 64, \mathrm{FOV}=240 \mathrm{~mm}$, measurements $/$ volumes $=121$, and scan time $=4: 06$

The images were analysed using the BrainLAB iPlan Cranial 3.0 software (BrainLAB AG, Feldkirchen, Germany). The fMRI data were preprocessed to correct for subject motion, and the DTI data were preprocessed to correct for distortions owing to eddy currents. Both the fMRI and DTI images were co-registered to the high resolution MPRAGE image. The fMRI activation patterns for the 4 motor tasks were displayed with a statistical significance of $p<0.001$. Fibre tractography was performed from a manually defined volume around the tumour. The minimum fibre length was set to $40 \mathrm{~mm}$; tracking was halted when the fractional anisotropy dropped below 0.15 . The tumour volume was semi-automatically demarcated on the MPRAGE images.

\section{Results}

Fig. 1 shows the rendered surface of the patient's brain from the MPRAGE image and the relative positions of the tumour (pink) and regions activated by the fMRI motor tasks. The motor region associated with the patient's right hand (red) lies immediately supero-lateral to the tumour. Note the clear symmetrical contralateral activation patterns in the cerebellum, and the reduced right foot activation compared with the left foot activation.

Fig. 2 shows the fibre tracts stemming from the manually defined region around the tumour (yellow box in Fig. 2b). Corticospinal tracts are shown to be pushed laterally by the tumour and also to extend around to the superior aspect of the tumour.

The patient was taken to theatre and the mass lesion was resected. According to the fMRI data, the tumour was located just deep to the motor cortex. A cortical incision through the gyrus directly overlying

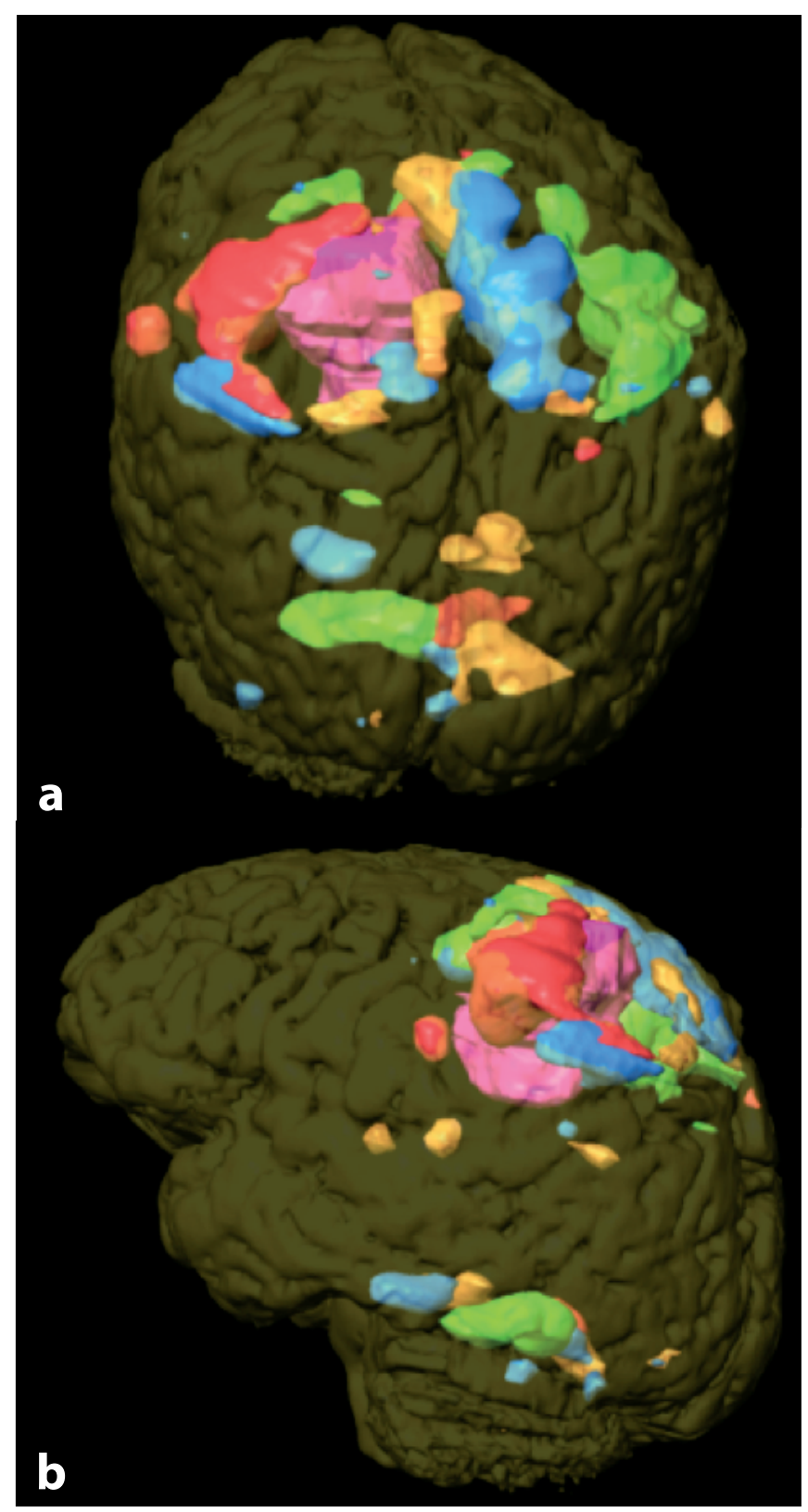

Fig. 1. Rendered brain surface showing the tumour (pink) and fMRI motor regions in (a) oblique-posterior, and (b) lateral views. Red is right hand/wrist/ forearm; blue is left hand/wrist/forearm; orange is right foot/toes; green is left foot/toes. Note the contralateral activation in the cerebellum. 

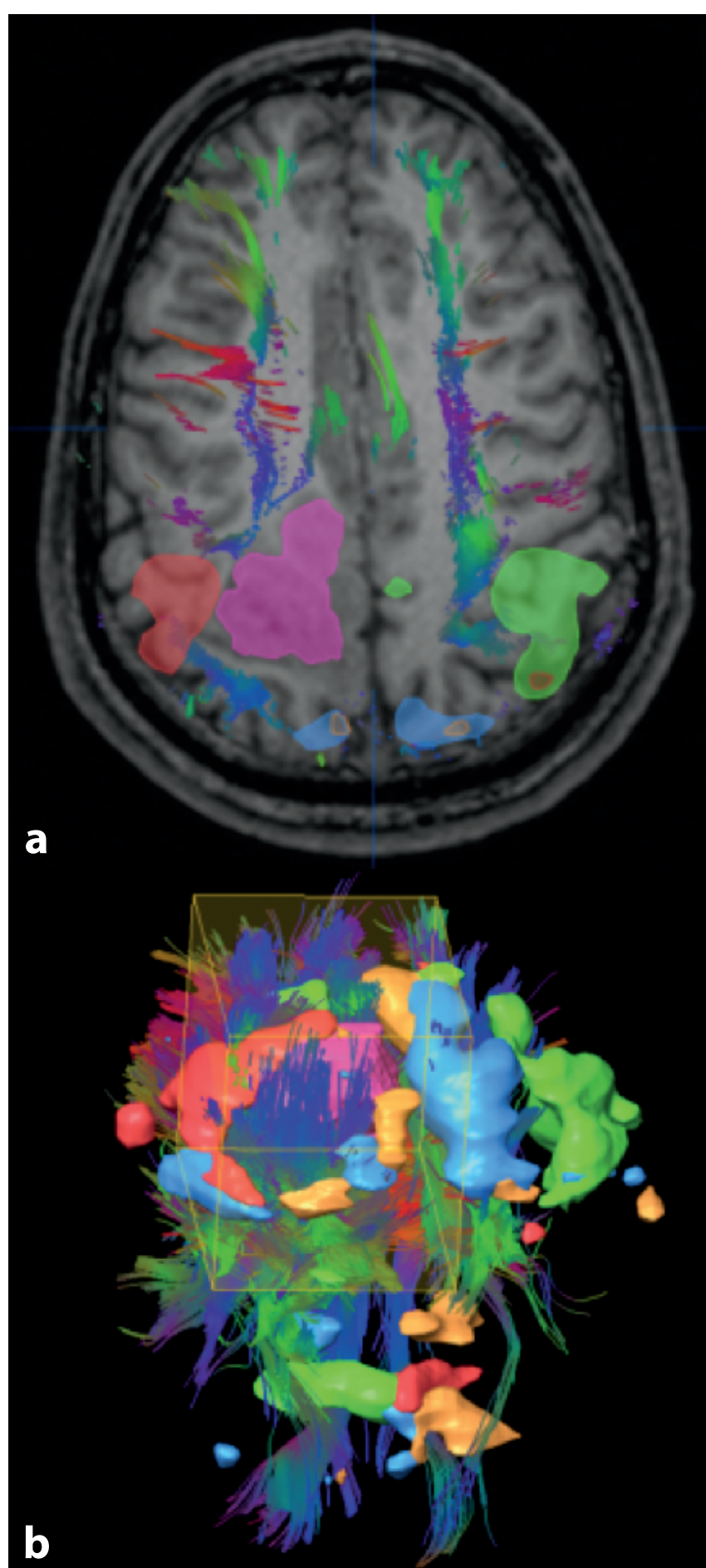

Fig. 2. DTI fibre tractography and $f M R I$ activation (a) overlaid onto an axial slice of the MPRAGE image at the level of the tumour, and (b) rendered in 3 dimensions. The corona radiata fibres in the left hemisphere in (a) are pushed laterally by the tumour. Some of the fibres deviated by the tumour can be seen in (b) to pass posteriorly and even superiorly to the tumour. The yellow box in (b) shows the manually-defined region used to seed the fibre tracking. The fibre colours correspond to the predominant fibre direction (green is anteroposterior; blue is crano-caudal; red is left-right).

the tumour would have injured the hand and foot area of the motor cortex. A more posterior approach through the sulcus just posterior of the motor strip was deemed safe. The motor cortex was identified clinically by evaluating the surface markings of the brain. Unfortunately, stereotaxis and cortical mapping were unavailable to further delineate the areas of interest. Surgery was uneventful and a good resection was achieved. Post-operatively, the patient was noted to have a right-sided drop foot $(0 / 5$ power) but the right hand function was intact. The foot drop improved and power was documented as $3 / 5$ at discharge on day 7 post surgery. The patient was able to leave the ward unassisted.

Histology showed the tumour to be an anaplastic astrocytoma. The patient was referred to our oncology department and received electron beam radiation therapy (EBRT). A follow-up scan showed no recurrence of tumour.

\section{Discussion}

The use of fMRI as a surgical planning tool continues to receive attention, as it could prove extremely valuable in many neurosurgical cases. Intra-operative cortical mapping (IOM), although invasive, can greatly aid the surgeon's ability to remove mass lesions in proximity to eloquent areas. Although fMRI has not yet proven to be a reliable replacement for $\mathrm{IOM},{ }^{8}$ it is a non-invasive and safe procedure that can aid in planning the surgical approaches needed for lesional resection by identifying eloquent areas. fMRI also has application in assessing the feasibility of tumor resection, ${ }^{3,13,14}$ and in identifying suitable candidates who might benefit from IOM..$^{15,16}$

The BOLD effect improves with the square of the strength of the magnetic field, therefore higher field strength MRI scanners are beneficial. ${ }^{17}$ However, susceptibility artifacts are also more pronounced at higher field strengths, which may result in incorrect spatial localisation. Although fMRI shows much promise in pre-surgical planning, processing and interpreting the results should be done with care. We advocate the following initial guidelines:

1. The output from each fMRI experiment is a statistical parametric map describing the probability of activation. It is important to realise that setting the threshold too high will include cortical activation that could be due to mere chance, and a low setting could lead to false negative findings, consequently missing eloquent cortical areas. The activation threshold should be based on the clinician's experience with the specific task, and should preferably be ascertained using a normal control sample.

2. fMRI uses blood flow as a surrogate marker for cortical activation; therefore blood vessels leading to the activated region may also cause signal changes.

3. Any tumour that interferes with the functional haemodynamic coupling could result in erroneous findings. The type of tumour may differentially affect the accuracy of the fMRI findings..$^{18}$ Other factors such as tumour extent, histology and presence of vasogenic oedema still need to be adequately explored at high field strengths (3T and above). ${ }^{14} \mathrm{~A}$ recent article $\mathrm{e}^{20}$ highlights such factors, where they report falsely localised fMRI results in the presence of an arteriovenous malformation in a paediatric neurosurgical case study.

4. The echo planar images (EPI) used in fMRI are prone to susceptibility artifacts; consequently, co-registration with the high-resolution structural MRI image will be imperfect, in particular near air-tissue interfaces (e.g. the inferior frontal regions). Activation patterns in these areas should be interpreted with care.

5. During an fMRI scan, numerous whole brain EPI volumes are 


\section{CASE REPORT}

acquired at intervals of $1-2$ seconds, making the technique susceptible to motion-related artifacts. The motion correction step in the fMRI pre-processing quantifies the head translations and rotations as a function of time, and the data should be discarded for translations more than about $2-3 \mathrm{~mm}$.

6. Quality assurance scans $s^{20}$ should be performed routinely to ensure that the long-term signal drift in the EPI images is within vendorspecified limits.

7. The presence of adjacent surgical clips or metallic implants from previous interventions also causes significant distortions and signal dropout in the EPI images, and will probably render the fMRI data useless.

8. The ability of fMRI to show reliable activation crucially depends on the task design and the appropriateness of the task for the target patient population, including factors such as age, education, culture and a host of other factors. Such factors might be even more relevant in low-income settings where patients' level of education could also influence task performance, which makes it crucial that each functional imaging centre rigorously pursues the validation of their own fMRI protocols tailored specifically to the eloquent region of interest, tumour type as well as patient population.

\section{Conclusion}

We have presented a case study where fMRI was used to identify the motor areas in relation to a tumour. The fMRI results provided useful information to guide resection of the tumour, and the surgery was successful. However, this was a relatively straightforward case using a well-established fMRI task. A detailed understanding of fMRI and appropriate precautionary measures should be taken when using fMRI to plan neurosurgical interventions.

Acknowledgements. We thank Siemens Medical Solutions South Africa; BrainLAB South Africa; and the staff at the Cape Universities Brain Imaging Centre.

1. Bandettini P. Functional MRI today. Int J Psychophysiol 2007;63:138-145

2. Ogawa S, Lee TM, Kay AR, Tank DW. Brain magnetic resonance imaging with contrast dependent on blood oxygenation. Proc Natl Acad Sci USA 1990;87:9868-9872.

3. Håberg A, Kvistad KA, Unsgård G, Haraldseth O. Preoperative blood oxygen level-dependent functional magnetic resonance imaging in patients with primary brain tumors: Clinical application and outcome. Neurosurgery 2004;54(4):902-915.

4. Hirsch J, Ruge MI, Kim KHS, et al. An integrated functional magnetic resonance imaging procedure for preoperative mapping of cortical areas associated with tactile, motor, language, and visual functions. Neurosurgery 2000;47(3):711-722

5. Blatow M, Reinhardt J, Riffel K, Nennig E, Wengenroth M, Stippich C. Clinical functional MRI of sensorimotor cortex using passive motor and sensory stimulation at 3 Tesla. JMRI 2011;34:429-437.

6. Biswal B, Yetkin FZ, Haughton VM, Hyde JS. Functional connectivity in the motor cortex of resting human brain using echo-planar MRI. Magn Reson Med 1995;34:537-541.

7. Mahdavi A, Houshmand S, Oghabian MA, et al. Developing optimized fMRI Protocol for clinical use: Comparison of different language paradigms. J Magn Reson Imaging 2011;34:413-419.

8. Giussani C, Roux F, Ojemann J, Sganzerla EP, Pirillo D, Papagno C. Is preoperative functional magnetic resonance imaging reliable for language areas mapping in brain tumor surgery? Review of language functional magnetic resonance imaging and direct cortical stimulation correlation studies. Neurosurgery 2010;66(1):113-120.

9. Suzuki M, Kitano H, Kitanishi T, et al. Cortical and subcortical activation with monaural monosyllabic stimulation by functional MRI Hearing Research 2002;163:37-45.

10. Lanting $\mathrm{CP}$, de Kleine $\mathrm{E}$, Bartels $\mathrm{H}$, van Dijk P. Functional imaging of unilateral tinnitus using fMRI. Acta Otolaryngol 2008;128(4):415-421.

11. Basser PJ, Mattiello J, LeBihan D. MR diffusion tensor spectroscopy and imaging. Biophysical J 1994;66:259-267.

12. Mori S, van Zijl P. Fiber tracking - principles and strategies: a technical review. NMR Biomed 2002;15:468480

13. Stippich C, Rapps N, Dreyhaupt J, et al. Localizing and lateralizing language in patients with brain tumors: Feasibility of routine preoperative functional MR imaging in 81 consecutive patients. Radiology;243(3):828-836.
14. Bizzi A, Blasi V, Falini A, et al. Presurgical functional MR imaging of language and motor functions: Validation with intraoperative electrocortical mapping. Radiology 2008;248(2):579-589.

15. Picht T, Kombos T, Gramm HJ, Brock M, Suess O. Multimodal protocol for awake craniotomy in language cortex tumour surgery. Acta Neurochir (Wien) 2005;148(2):127-138.

16. Sherman JH, Hoes K, Marcus J, Komotar RJ, Brennan CW, Gutin PH. Neurosurgery for brain tumors update on recent technical advances. Curr Neurol Neurosci Rep. 2011 Jun.;11(3):313-319.

17. Li S-W, Wang J-F, Jiang T, et al. Preoperative 3T high field blood oxygen level dependent functional magnetic resonance imaging for glioma involving sensory cortical areas. Chin Med J 2010;123(8):10061010

18. Jiang Z, Krainik A, David O, et al. Impaired fMRI activation in patients with primary brain tumors NeuroImage 2010;52(2):538-548.

19. Juenger $\mathrm{H}$, Ressel V, Braun C, et al. Misleading functional magnetic resonance imaging mapping of the cortical hand representation in a 4-year-old boy with an arteriovenous malformation of the central region. J Neurosurg Pediatr 2009;4(4):333-338

20. Weisskoff RM. Simple measurement of scanner stability for functional NMR imaging of activation in the brain. Magn Reson Med 1996;26:643-645 